\title{
What you need to know when you go with the flow: pitfalls in the use of flow cytometry
}

Flow cytometry is a useful technique for determining the molecules present on, or sometimes within, single cells in suspension. The molecules of interest are targeted with a fluorochrome label, which is usually delivered by means of antibody, and are detected as the cells pass in single file through a fluorescence detector. Flow cytometry is most frequently used for leucocyte subset analysis, and technical training focuses accordingly on the determination of antigens which are strongly expressed-for example there are approximately $10^{4} \mathrm{CD} 4$ molecules per positive lymphocyte. However, appreciation of more complex aspects of flow cytometry may be lacking, which can cause problems in its application as a research tool. For example, in a long term study to compare the expression of an antigen in patient and control groups, how do we know that results are reproducible over time? If an antigen is weakly expressed, how do we decide how many cells are positive and how many negative? How do we calculate how many antigens per cell? To add to these problems, quality control, the sine qua non of most laboratory practice, has been slow to develop, because of the difficulty of obtaining and distributing suitable material, and this may be part of the reason why studies with no apparent attempt to standardise results manage to reach the literature. As flow cytometry is being increasingly used by rheumatologists, particularly in the search for cellular abnormalities underlying rheumatic diseases, it is necessary to make sure that interesting studies are not undermined by poor design. We discuss three key areas which should be considered when planning flow cytometric studies: reproducibility, amplification, and data analysis.

Many factors can affect the reproducibility of a flow cytometric method. The stability of the antigen itself must be considered-that is, how it is affected by storage, preparation, and fixation. ${ }^{12}$ Saturating dilutions of antibody must be determined using titration experiments. If there is a choice between using a fluorochrome conjugated antibody to the antigen of interest (direct method) or an unlabelled antibody followed by an amplification/detection step (indirect method), the merits of each method should be understood. Amplification is useful for low density antigen, ${ }^{3}$ but, for comparative studies, adding extra variables increases the variance of each sample distribution and therefore increases the likelihood of a type II statistical error. ${ }^{4}$ For direct tests, isotype matched labelled antibodies to irrelevant antigens should be used at the same concentrations as test antibodies to control for non-specific binding. Indirect tests should be controlled using irrelevant isotype matched primary antibody. Particular care is required in studies combining an indirect method for one antigen with a direct method for a second antigen on the same sample. Free binding sites on the second layer antibody labelled with fluorochrome 1 could bind directly to the conjugated antibody labelled with fluorochrome 2 . This can be minimised by blocking free binding site on the second layer antibody with normal serum from the appropriate species. Controls for each antigen singly should be used to confirm that there is a similar degree of positivity in the single stained and double stained samples.

Variability in the fluorescence signal, as a result of instability of the antibody or fluorochrome, variability in the assay, or instrument drift, may not be crucial for a CD4 count, but need to be considered in studies that compare small values or values over time. An assay can be standardised with a number of the stable, fluorescent beads on the market. ${ }^{5-8}$ (However, although fluorescent beads are suitable for standardising results, they may not be suitable for calibrating fluorescent molecule density. We (unpublished data) and others ${ }^{5}$ have noted batch to batch variation in calibrated beads, which compounds the uncertainty already associated with spectrophotometric calibration. ${ }^{6}$ ) The use of frozen or lyophilised cells for quality control may give results which are too variable to assure individual patient results in the health service, but may still be worth using for analysis of trends in instrument and antibody behaviour in a long term research study. ${ }^{178}$ Moreover, with standard formaldehyde fixation of leucocytes, many antigens are stable at $4^{\circ} \mathrm{C}$ for at least seven days and can be repeatedly analysed to assess accuracy of standardisation or instrument drift over time. ${ }^{4} 7$

Amplification of the fluorescence signal is logarithmic in most published studies, which again reflects the needs of the health service laboratory determining the presence or absence of antigen. However, logarithmic amplification is not always appropriate. ${ }^{9}$ Logarithmic data have to be antilogged before non-specific fluorescence can be subtracted, and log to linear transformation is not straightforward, particularly at the lower end of the scale, which introduces inaccuracies. ${ }^{10} \mathrm{~A}$ further problem with logarithmic amplification is that as the number of fluorescence signals per cell increases, each channel records an increasingly compressed range of values, with a resultant loss of precision. Linear amplification can be combined with use of the base gain control in order to extend the range of values which can be obtained and is particularly valuable when discriminating between two relatively strong signals (figure).

Software packages now offer a choice of statistical parameters for quantifying antigens and the appropriate use of mean, percentiles and mode should be considered. ${ }^{11} 12$ Before starting a study, it is worth giving some thought to the form in which results will be obtained, with a view both to presenting results intelligible to the non-specialist, and to facilitating independent verification by other laboratories. This is not achieved by the all too common reporting of mean fluorescence in arbitrary units on a logarithmic or unspecified scale. Variances and differences between patient groups which appear small on a log scale are much greater on a linear scale. The figure shows that the $70-80$ fold increase of fluorescence on the brightest (B5) compared with the dimmest (B1) bead is displayed logarithmically as merely an approximate doubling of the channel numbers on the FACScan. There is also a common misconception that all analysis programs convert logarithmically acquired data into linear form in order to arrive at 'mean fluorescence intensity' units (some do; some don't). It is essential for authors to understand the methodology of their own software, and also to inform the reader. For ease of understanding, we would prefer to see all logarithmic data converted to a linear scale.

The figure illustrates an analysis of fluorescent beads on both logarithmic and linear scales. Data were collected on a single tube of fluorescent beads (Dako fluorospheres) with a FACSan (Becton Dickinson) using linear and 


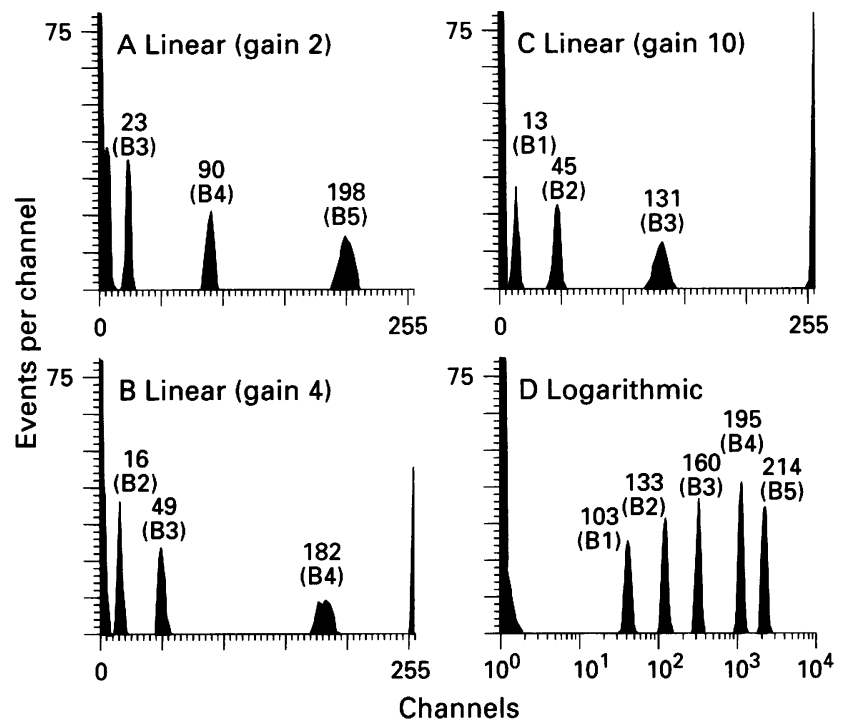

Use of linear gain control to increase the range of values which can be obtained with linear amplification. $A-C$ : Linear amplification; $D$ : logarithmic amplification. Fluorescent beads are labelled BO for blank and B1-B5 in ascending order of brightness. The gain setting for each plot is shown, and each peak is labelled with the appropriate bead number and peak channel number. Note that values must be multiplied by $10 \times$ the reciprocal of the gain in order to compare values obtained for the same bead at different settings.

logarithmic amplification. The beads had been calibrated by a previously described system using iodine-125-fluorescein isothiocyanate dual labelled antibody, ${ }^{13}$ such that the brightest fluorescence (B5 on the histograms) was calculated to be equivalent to $1.7 \times 10^{5}$ bound fluorochrome molecules. The dimmest bead (B1 on the histograms) is approximately 75 -fold less bright than the brightest, but both beads can be acquired from the same tube and at the same voltage by altering the gain setting.

Another common, but poor, practice is the quantitation of weakly expressed antigens as percent positive by setting a cut off point on the 'tail' of a histogram, as discussed previously by Zola. ${ }^{3}$ This author recommended the application of more sensitive labelling, but it is also possible to increase sensitivity at the analysis stage, for example by quantifying the mean or percentile difference between test and blank fluorescence (using a linear amplifier) and expressing results as an overall difference in fluorescence intensity per cell rather than as a percentage of positive cells. It is also worth considering the use of cluster analysis to estimate the percentage of positive cells. ${ }^{14}$
There are frequent abnormalities of relative and of absolute leucocyte subset numbers in rheumatic diseases, and there is therefore a potential pitfall in the interpretation of a finding that a given molecule is abnormally distributed. Unless care is taken to compare like with like subset, an unusual distribution of a molecule could simply reflect an abnormal distribution of the subsets on which that molecule is found. To give a hypothetical example, a finding of comparatively low lymphocyte CD25 expression in a group of patients with low CD4:CD8 ratios would probably merely reflect the fact that CD4 lymphocytes tend to express far more CD25 than CD8 lymphocytes.

We hope that increased awareness of the issues involved in flow cytometric quantitation, particularly the need for reproducible methodology, will facilitate the more effective application of this powerful technique to rheumatological problems.

Department of Immunology,

MONICA PALLIS

University Hospital,

R ADRIAN ROBINS

Queen's Medical Centre,

Nottingham NG7 2UH

United Kingdom

1 Caldwell C W, Maggi J, Henry L B, Taylor H M. Fluorescence intensity as a quality control parameter in clinical flow cytometry. Am $\mathcal{f}$ Clin Pathol 1987, 88: $447-56$.

2 McCarthy D A, Macey M G, Cahill M R, Newland A C. Effect of fixation on quantification of the expression of leucocyte function-associated on quantification of the expression of le

$3 \mathrm{Zola} \mathrm{H}$. Studies of cytokine receptor expression by cells of the immune system-a waste of time? Immunol Today 1992; 13: 419-20.

4 Traill K N, Böck G, Winter U, Hilchenbach M, Jürgens G, Wick G. Simple method for comparing large numbers of flow cytometry histograms exemplified by analysis of the CD4 (T4) antigen and LDL receptor on human peripheral blood lymphocytes. $\mathcal{f}$ Histochem Cytochem 1986; 34: 1217-21.

5 Bell A, Shenton B K. Quality control in flow cytometry. Cytometry 1994; 7 (suppl): 38 .

6 Vogt R F, Cross G D, Henderson L O, Phillips D L. Model system evaluating fluorescein-labelled microbeads as internal standards to calibrate fluorescence intensity on flow cytometers. Cytometry 1989; 10: brate fluore

7 Pallis M, Robins A, Powell R. Quantitative analysis of lymphocyte CD11a using standardised flow cytometry. Scand f Immunol 1993; 38: 559-64.

8 Lavabre-Bertrand T, Janossy G, Ivory K, Peters R, Secker-Walker L, Porwitt-Macdonald A. Leukemia-associated changes identified by quantitative flow cytometry: I. CD10 expression. Cytometry 1994; 18: 209-17.

9 Gandler W, Shapiro H. Logarithmic amplifiers. Cytometry 1990; 11: 447-50.

10 Schmid I, Schmid P, Giorgi J V. Conversion of logarithmic channel numbers into relative linear fluorescence intensity. Cytometry 1988; 9: 533-8.

11 Vogt R F, Henderson L O, Ethridge S F, Huang E Y, White J T, Meredith N K. Lymphocyte immunophenotyping with extended quantitative analysis of listmode files for epidemiologic health studies. $A n n N Y A c a d$ Sci 1993; 677: 462-4.

12 Coder D M, Redelman D, Vogt R F. Computing the central location of immunofluorescence distributions: logarithmic data transformations are immunofluorescence distributions: logarithmic da

13 Roe R, Robins R A, Laxton R R, Baldwin R W. Kinetics of divalent monoclonal antibody binding to tumour cell surface antigens using flow cytometry: standardisation and mathematical analysis. Mol Immunol 1985; 22: 11-21

14 Bakker Schut T C, De Grooth B G, Greve J. Cluster analysis of flow cytometric listmode data on a personal computer. Cytometry 1993; 14: 649-59. 